\title{
One-pot Synthesis of Octyne-Ruthenium on Carbon Nanoparticles
}

\author{
Kanda Wongwailikhit ${ }^{1, a}$, Rawewan Chaisinchareon ${ }^{2}$ and Shaowei Chen ${ }^{2}$ \\ ${ }^{1}$ Department of Chemistry, Faculty of Science, Rangsit University, Patumtani, Thailand \\ ${ }^{2}$ Department of Chemistry and Biochemistry, University of California, Santa Cruz, US
}

\begin{abstract}
The attempts to manipulate ruthenium nanoparticle by the passivation of $\pi$ bonds linkage is of interest for many years. That is the way to enhance its optical properties and fluorescence characteristics which can promote the usage for sensor application. Other view, the usage of carbon nanoparticle is governed in many aspects including its fluorescence properties. Therefore, the combination between those two valued nanoparticles was set by conducting the simple synthesis method. With the as-prepared carbon nanoparticles, all other reagents (ruthenium (III) chloride, octyne and Sodium borohydride) were mixed in the same batch. The ratio of carbon substrate, ruthenium (III) chloride and octyne was 10: 1: 3. The particle yielded was then purified and subjected to characterize using some spectroscopy techniques including photoluminescence. The results showed that size of carbon particle before and after ruthenium deposition were 5.0 and 6.3 nanometers, respectively. Octyne was coordinated self-assembly on the ruthenium surface which was 8.1 nanometers in diameter. Moreover, octyne-protected ruthenium on carbon nanoparticles showed the remarkably increasing of fluorescence Intensity. Therefore, the functionalization of carbon nanoparticle with octyne-ruthenium can be a promising strategy to develop a novel complex of ruthenium.
\end{abstract}

\section{Introduction}

Recently, many reports have been generally successfully prepared alkyl vinylidene -linkage to ruthenium core using the special coordinating ligand for example terminal alkyne [1-5] or some vinyl aromatic [6-7]. It is confirmed that alkyne fragments around the metal core do not only functions as protecting ligands promoting the stability of the nanoparticles but also enhances some optical and fluorescence characteristic via the pi-bond interaction between methylene and metal core[8]. It should be noted that alkynes (and alkenes as well) have been used rather extensively as coordinating ligands in the preparation of varied transition metal complexes [9]. With two $\pi$ bonds, alkynes can form stable complexes in which they bridge two metal centers. Then alkyne can donate total four electrons which support the exploitation of metal-carbon covalent linkages for nanoparticle surface functionalization [10]. The conjugated interfacial bonds can extend the intraparticle charge delocalization between particle-bound functional moieties, leading to increase optical and electronic properties [11].

\footnotetext{
${ }^{\text {a }}$ Corresponding author: kanda@rsu.ac.th
} 
This work, we try to develop the idea of using carbon nanoparticles (CPNs) as the working core instead of pure ruthenium nanoparticle. It is well known that carbon nanoparticle is enriched with carbonyl group which occurred simultaneously with the formation of the CNPs, and effective surface states for luminescence such and used as chemical sensing, biological labeling, and optoelectronics [12]. Herein, the ruthenium metal was attempted to deposit onto CPNs surface and then covered the outer-shell with alkyne. The process was conducted in one-pot synthesis and testified whether the deposition was be succeeded. The properties of the products were determined with some spectrophotometric methods including the photoluminescence properties.

CPNs was prepared from soot with the same direction reported previously [13]. The nanosized carbon particles synthesized composed of $\mathrm{sp}^{2}$ graphitic carbons in the crystalline core with good photoluminescence in the visible range. Moreover, it was reported that and the surface of carbon was plenty of oxygenated functional moieties. Ruthenium chloride and sodium borohydride were used as source of metal ion and reducing agent, respectively. Octyne was chosen to be the functionalized ligand onto ruthenium surface.

\section{Chemicals}

Ruthenium chloride $\left(\mathrm{RuCl}_{3}, 99+\%\right.$, ACROS), 1-octyne (Alfa Aesar, 98\%), Nitric acid $\left(\mathrm{HNO}_{3}, 69.8 \%\right.$, Fisher), sodium carbonate $\left(\mathrm{Na}_{2} \mathrm{CO}_{3}, 99+\%\right.$, Aldrich), methanol $\left(\mathrm{CH}_{3} \mathrm{OH}\right.$, Fisher Scientific] and sodium borohydride $\left(\mathrm{NaBH}_{4}, 99 \%\right.$, ACROS) were all used as received. Water was supplied by a Barnstead Nanopure Water System $(18.3 \mathrm{M} \Omega 3 \mathrm{~cm})$.

\section{Methodologies}

\subsection{Synthesis of Carbon nanoparticles}

Carbon Nanoparticles was prepared in the same procedure of published articles [13-14]. Briefly described as, Carbon soot was collected on the inside wall of a glass beaker by placing the beaker upside-down above the flame of a natural gas burner. After being washed with acetone and dried at $110^{\circ} \mathrm{C}, 100 \mathrm{mg}$ of the soot was then refluxed in $10 \mathrm{~mL}$ of $5 \mathrm{~mol} / \mathrm{L} \mathrm{HNO}_{3}$ at $140^{\circ} \mathrm{C}$ for $12 \mathrm{~h}$. After the mixture was cooled to room temperature, the brownish solution was neutralized by $\mathrm{Na}_{2} \mathrm{CO}_{3}$ and loaded into dialysis bags (cutoff molecular weight 8000-14,000) for dialysis against ultra-pure water for three days to purify the CNPs. Then CNPs was dialyzed against ultra-pure water for 3 days. Water in dialysis process was replenished every day. CNPs were collected by centrifugation at 16,000 rpm. The precipitation was dried under nitrogen flow and dispersed again in ultra-pure water. Big particles were removed using ultrafiltration till brown solution of CNPs was obtained. This solution was kept at $4^{\circ} \mathrm{C}$ until use.

\subsection{Synthesis of Alkyne-Ruthenium on Carbon nanoparticles}

Octyne-ruthenium was deposited on CPNs by mixing $\mathrm{RuCl}_{3}, \mathrm{NaBH}_{4}$, and $\mathrm{CNPs}$ at the ratio of 3:3:10 in pure water. Briefly describe, $10 \mathrm{mg}$ of CNPs was mixed with $1 \mathrm{mg} \mathrm{RuCl}{ }_{3}$ in $10 \mathrm{~mL}$ of water and stir over night. Octyne was added at the mole ratio between $\mathrm{RuCl}_{3}$ and octyne of 1:3. Then, a calculated amount of $\mathrm{NaHB}_{4}$ was added in a slow drop-wise fashion. The solution color changed gradually from light brown to dark brown, signifying the formation of Ruthenium under reduction reaction. The resulting solution was allowed to stir at room temperature over $3 \mathrm{~h}$. After the reaction, the carbon-supported Ruthenium nanoparticles were treated with methanol and separate over the ultracentrifuge. The particles were found to be readily soluble in typical non-polar solvents such as toluene, tetrahydrofuran, chloroform, and dichloromethane, and not soluble in polar solvents such as alcohols, acetone, and acetonitrile. Spectroscopic measurements were then carried out to examine the product. A set of experiment was also run with the same procedure except adding octyne. The 
resulting particle was used to compare the result between octyne-protected ruthenium on carbon nanoparticle and bare ruthenium on carbon nanoparticle

\section{Results}

As mention above, aim of our work was to prove if the synthesis process was able to short with one step of relevant mixtures. The target yield was the nanoparticle of ruthenium metal deposited on CPNs (denoted as $\mathrm{C} / \mathrm{Ru}$ ) and octyne was linked on top of ruthenium surface with the $\pi$-covalent bond (denoted as $\mathrm{C} / \mathrm{Ru} / \mathrm{Oct}$ ). Below implied the results which illustrating the success of the production.

The formation of $\mathrm{C} / \mathrm{Ru} / \mathrm{Oct}$ nanoparticles was first verified by $\mathrm{UV}$-visible analysis measurements. Figure 1 (a) shows the UV-visible absorption spectrum of carbon nanoparticles in water $(0.1 \mathrm{M})$. The particles exhibited a major absorption peak at around $200 \mathrm{~nm}$ ascribing to the $\mathrm{p}-\mathrm{p}^{*}$ electronic transitions of internal $\left(\mathrm{sp}^{2}\right)$ graphitic carbons. Figure 1 (b) responses the changes of carbon nanoparticle spectrum to the featureless exponential decay profile anticipating for nanosized $\mathrm{Ru}$ particles [15]. This suggested the success of the ruthenium deposition. Figure 1(c) depicts a UV-vis spectrum of the $\mathrm{C} / \mathrm{Ru} /$ Oct nanoparticles prepared. It can be seen that the $\mathrm{Ru}$ nanoparticles were all well dispersed without apparent aggregation, suggesting effective passivation of the alkyne ligands on the Ru particle surfaces.

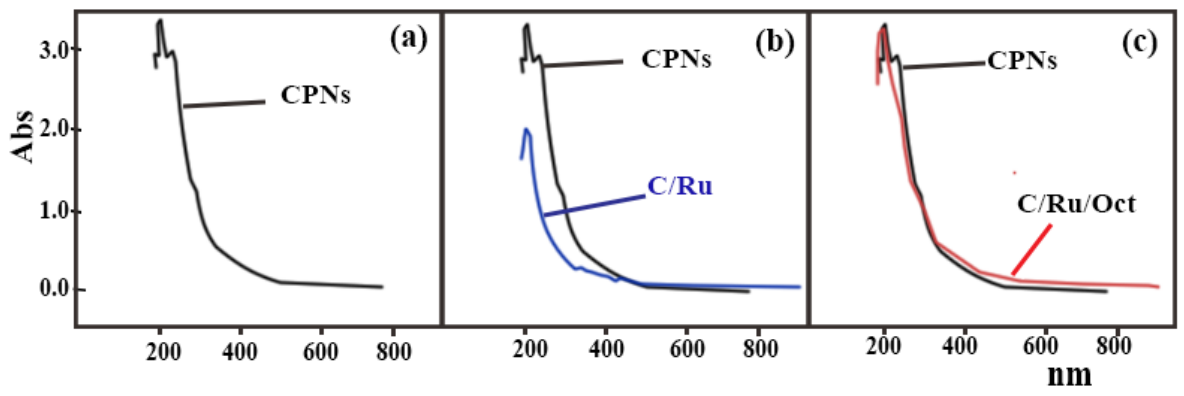

Figure 1. The UV-visible absorption spectrum of products in water $(0.1 \mathrm{M})$, (a) $\mathrm{CPNs}(\mathrm{b}) \mathrm{C} / \mathrm{Ru}$ (c) $\mathrm{C} / \mathrm{Ru} / \mathrm{Oct}$

All particles were investigated using particle size analysis and the results were shown in figure 2 . It was corresponding to the results interpreting by UV-visible spectrum. Figure 2 (a) showed the average of particle size of CPNs about $5.0 \mathrm{~nm}$. After the ruthenium deposition, $\mathrm{C} / \mathrm{Ru}$, size of particle was raised to about $6.3 \mathrm{~nm}$ (figure 2 (b)). Finally with the passivation of octyne, C/Ru/Oct, size of average particle was increased to $8.1 \mathrm{~nm}$ (figure 2 (c)) which suggesting the effective passivation of the octyne ligands on the ruthenium surfaces. This suggested that thickness of ruthenium layer plus octyne layer was about $3.1 \mathrm{~nm}$. It was relatively close to the octyne passivated on ruthenium nanoparticle synthesized by Chen, W. et. al. [16] which was 2-3 nm sized average. Herein, it was reasonable to conclude the successful production of our process.

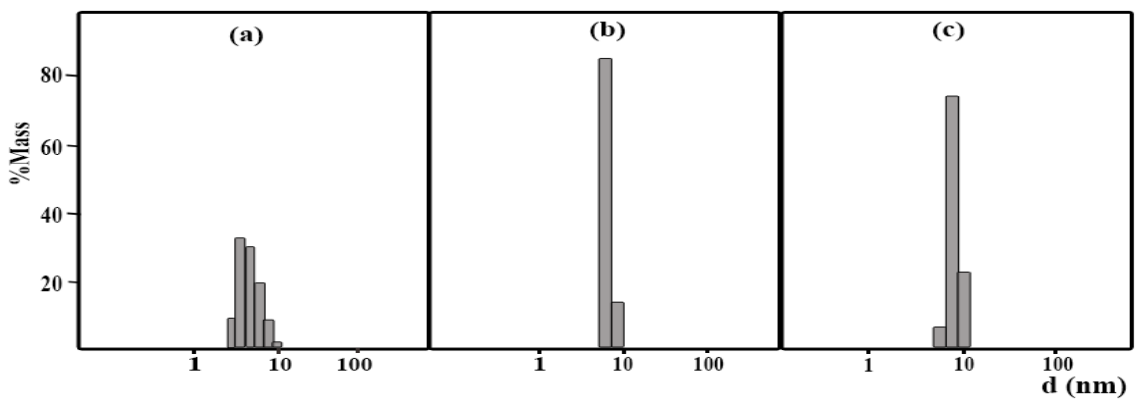

Figure 2. Particle size analysis results obtained from (a) $\mathrm{CPNs}$ (b) $\mathrm{C} / \mathrm{Ru}$ (c) $\mathrm{C} / \mathrm{Ru} / \mathrm{Oct}$ 
The structure of pure carbon nanoparticles and $\mathrm{C} / \mathrm{Ru} /$ Oct were then examined by FTIR spectrum and compare with CPNs as shown in figure 3.

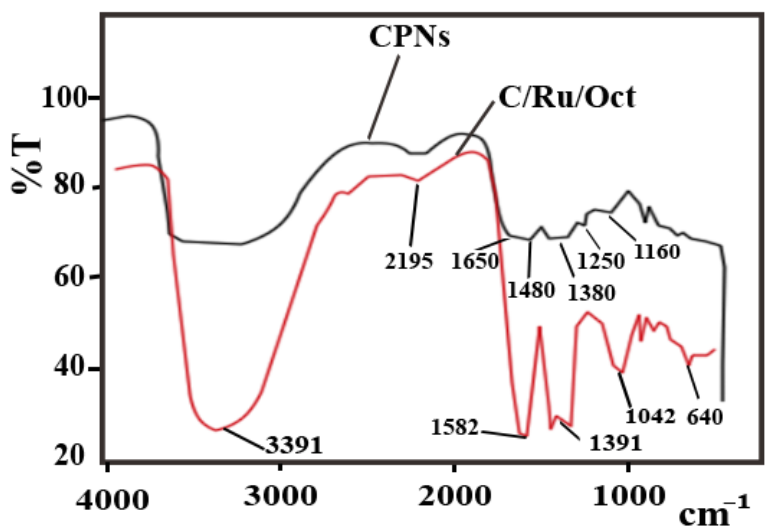

Figure 3. FTIR spectra of CPNs and $\mathrm{C} / \mathrm{Ru} / \mathrm{Oct}$

For the CPNs, a characteristic peak at $1160 \mathrm{~cm}^{-1}$ was assigned to $\mathrm{C}-\mathrm{C}$ bonds. Also, the spectrum showed the carbonyl characteristic broad peak at $1650 \mathrm{~cm}^{-1}$, which was assigned to the carbonyl group from quinine or ring structure. [17] Moreover, at $1480 \mathrm{~cm}^{-1}$ suggesting $-\mathrm{C}-\mathrm{H}$ and $1380 \mathrm{~cm}^{-1}$ showed the $-\mathrm{C}-\mathrm{O}$ - and $1250 \mathrm{~cm}^{-1}$ showed the $\mathrm{O}=\mathrm{C}-\mathrm{OH}$ which was the special characteristic of carbon nanoparticles synthesized using soot [18].

For $\mathrm{C} / \mathrm{Ru} / \mathrm{Oct}$, a characteristic peak at $1582 \mathrm{~cm}^{-1}$ was assigned to $\mathrm{C}=\mathrm{C}$ bond. This should be said that $\mathrm{C}=\mathrm{C}$ band was such strong and showed the plenty of $\mathrm{C}=\mathrm{C}$ bond after passivation with Octyne. The broad band centered around $3500 \mathrm{~cm}^{-1}$ most likely arose from residual water. The $-\mathrm{C} \equiv \mathrm{C}-$ vibrational stretch of alkynes was shown at $2195 \mathrm{~cm}^{-1}$ which typically observed for monomeric alkynes as a weak band within $2260 \mathrm{~cm}^{-1}$ [19]. This shift was responsible to the complex between metal and alkyne [20]. The absent of peaks at $1250 \mathrm{~cm}^{-1}$ suggested that $\mathrm{O}=\mathrm{C}-\mathrm{OH}$ was reduced in the synthesis process and form R-O-H or R-O-R which was absorbed at $1042 \mathrm{~cm}^{-1}$. The strong peak at $1390 \mathrm{~cm}^{-1}$ showed the increase up of $-\mathrm{C}-\mathrm{H}$ bond from octyne tail [21]. This suggested that the octynyl fragments were indeed bound onto the metal surface.

Further supporting evidence was manifested in the photoluminescence measurements and shown in figure 4 .

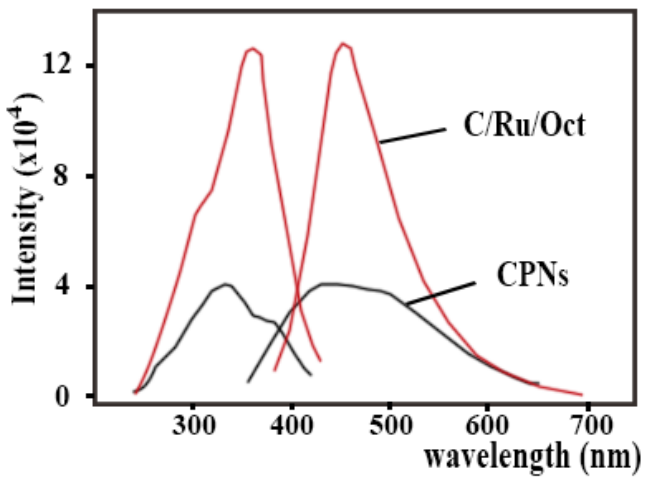

Figure 4. Excitation (left) and emission (right) spectra of CPNs (black) and $\mathrm{C} / \mathrm{Ru} / \mathrm{Oct}$ (red) at $25^{\circ} \mathrm{C}$. The particle concentration was $0.1 \mathrm{mg} / \mathrm{mL}$.

Figure 4 showed the excitation and emission spectra of the corresponding carbon nanoparticles before and after octyne-protected Ruthenium treatments in. It illustrated that the excitation and emission peak energies had practically changed from $\lambda \mathrm{ex}=330 \mathrm{~nm}$ and $\lambda \mathrm{em}=430-500 \mathrm{~nm}$ (440) to 
$\lambda \mathrm{ex}=360 \mathrm{~nm}$ and $\lambda \mathrm{em}=450 \mathrm{~nm}$. This suggested a different radiative decay pathway for the photoexcited electrons in the original CPNs and treated particles. In sharp contrast, the photoluminescence intensity exhibited drastic enhancement of more than the original ones. This significant improvement in the emission intensity was most likely due to the success of the deposition of ruthenium and the passivation of octyne over on ruthenium. Additionally, it can be seen that the adsorption peaks became narrower and contributed to the small red shift after treatment on metal nanoparticles [22]. The well-defined excitation and emission peaks shown at 360 and $450 \mathrm{~nm}$, respectively were corresponding to the previous studies of ruthenium nanoparticles passivated by 1dodecyne [7] and 1-octyne [16]. The high intensity of photoluminescence observed with the $\mathrm{C} / \mathrm{Ru} / \mathrm{Oct}$ nanoparticles strongly suggests that intraparticle charge delocalization took place as a result of the strong $\mathrm{Ru}=\mathrm{C}$ interfacial bonding interactions. Again, the successful incorporation of 1 -octyne onto the nanoparticle surface further confirms the formation of a $\mathrm{Ru}$-vinylidene interfacial linkage with nanoparticles.

\section{Conclusions}

The attempt to use CPNs as the center core for depositing ruthenium metal and the outer surface was protected with octyne was successful with a very simple hydrothermal method. CPNs was prepared from soot and mixed with octyne, $\mathrm{RuCl}_{3}$ and sodium borohydride as the reducing agent. The asprepared product was collected, purified and subjected to characterize some properties. The results shown that the one pot synthesis could produce the novel nanoparticles having the carbon nanoparticle core with ruthenium plated on the surface and the outer most shell was the vinylidene linkage of terminal octyne. Moreover, because of the specific photoluminescence properties, the nano-complex of carbon-ruthenium protected with octyne could be a promising particle for supporting the novel sensor technology.

\section{References}

1. X. Kang, S. Chen, Nanoscale. 4, 4183 (2012)

2. H. Sung, T. Her, W. Su, C. Cheng, Molecules. 17, 8533 (2012)

3. N.T. Lucas, M.P. Cifuentes, L.T. Nguyen, M.G. Humphrey, Cluster Sci. 12, 201 (2001)

4. C. Chen, H. Tong, C. Hsu, C. Lee, Y. Fong, Y. Chuang, Y. Lo, Y. Lin, Y. Wang, Organometallics. 28, 3358 (2009)

5. H. Kopf, C. Pietraszuk, E. Hübner, N. Burzlaff, Organometallics. 25, 2533 (2006)

6. V. Dragutan, I. Dragutan, Platinum Metals Rev. 48, 148 (2004)

7. X. Kang, N.B. Zuckerman, J.P. Konopelski, S. Chen, J. Am. Chem. Soc. 134, 1412 (2002)

8. W. Chen, S. Chen, F. Ding, H. Wang, L.E. Brown, J.P. Konopelski, J. Am. Chem. Soc. 130, $12156(2008)$

9. Structure and bonding of alkyne complex. Available at http://www.ilpi.com/organomet/ alkyne.html

10. W. Chen, L.E. Brown, J.P. Konopelski, S. Chen, Chem. Phys. Lett. 471, 283 (2009)

11. W. Chen, N.B. Zuckerman, J.W. Lewis, J.P. Konopelski, S. Chen, J. Phys. Chem. C.113, 16988 (2009)

12. M.S. Dresselhaus, G. Dresselhaus, Nanostruct. Mater. 9, 33 (1997)

13. B.R. Selvi, D. Jagadeesan, B.S. Suma, G. Nagashankar, M. Arif, K. Balasubramanyam, M. Eswaramoorthy, T.K. Kundu, Nano Lett. 8, 3182 (2008)

14. L. Tian, D. Ghosh, W. Chen, S. Pradhan, X. Chang, S. Chen, Chem. Mater. 21, 2803 (2009).

15. X. Kang, S. Chen, Nanoscale. 4, 4183 (2012)

16. W. Chen, N.B. Zuckerman, X. Kang, D. Ghosh, J.P. Konopelski, S. Chen, J. Phys. Chem. C,114, $18146(2010)$

17. Z. Yunusa, M.N. Hamidon, A. Ismail, M.M. Isa, M.H. Yaacob, S. Rahmanian, S.A. Ibrahim, A.A. Shabaneh, Sensors. 2015, 15, (2015) 
18. X. Sun, J. He, Y. Meng, L. Zhang, S. Zhang, X. Ma, S. Dey, J. Zhaoc, Y. Lei, J. Mater. Chem. A, $4,4161(2014)$

19. Infrared spectroscopy table. Available at; http://www.ochemonline.com/Infrared_spectroscopy_ absorption_table

20. N.J. Long, C.K. Williums, Angewandte Chemie. 42, 2586 (2003)

21. X. Kang, N.B. Zunkerman, J.P. Konopelski, S. Chen, J.Am.Chem. Soc. 134, 1412 (2012)

22. Chen, S. Pradhanx, S. Chen, Nanoscale. 3, 2294 (2011) 